\title{
Edema de pulmón precipitado por amlodipino
}

\author{
Pulmonary Edema Triggered by Amlodipine
}

\author{
Teresa Chapela Castaño ${ }^{1}$, José Luis Penas Ríos ${ }^{2}$ \\ ${ }^{1}$ Centro de Salud Elviña, ${ }^{2}$ Servicio de Medicina Interna. Complexo Hospitalario Universitario A Coruña. SERGAS. A Coruña
}

\begin{abstract}
Resumen
Múltiples fármacos pueden causar edema agudo de pulmón no cardiogénico. Establecer esta relación causal resulta muy difícil en muchos casos debido a la escasa incidencia documentada con algunos fármacos y a que la sintomatología no es específica. Si bien el edema periférico es un efecto secundario frecuente del amlodipino, solamente se han publicado en la literatura dos casos de edema pulmonar causado por amlodipino y ambos ocurrieron en el contexto de dosis supraterapéuticas. Se describe un caso excepcional de edema pulmonar probablemente inducido por amlodipino administrado a dosis terapéutica y se discute el posible mecanismo fisiopatológico.
\end{abstract}

Palabras clave: Amlodipino. Edema pulmonar. Efecto secundario.

\section{Introducción}

Amlodipino es un inhibidor de la entrada de iones calcio del grupo de las dihidropiridinas (antagonista del calcio) que impide el paso de los iones de calcio a través de la membrana celular en el músculo cardíaco y en el músculo liso vascular, provocando una vasodilatación por relajación directa ${ }^{1}$. Empleado en el tratamiento de la hipertensión arterial y la angina, entre los efectos secundarios más frecuentes $(\geq 1 / 100 \mathrm{a}<1 / 10)$ destacan la cefalea, la rubefacción 0 flushing y el edema. Por el contrario el edema pulmonar no consta como reacción adversa en la ficha técnica ${ }^{1}$, aunque sí se menciona un artículo de Packer et al, en el que se concluye que amlodipino no incrementa la morbilidad ni la mortalidad en pacientes con insuficiencia cardíaca grave y en el que se considera bajo (5\%) el riesgo de edema pulmonar atribuible a amlodipino en esta población². Se realizó una búsqueda mediante MEDLINE (1966-Diciembre 2012) de artículos publicados en lengua inglesa empleando como palabras clave "amlodipine" $y$ "pulmonar y edema" o "acute lung injury" o "heart failure". Solo se encontraron 2 casos de edema pulmonar en relación con amlodipino, en el contexto de sobredosificación el primero ${ }^{3}$ y de envenenamiento el segundo ${ }^{4}$.

\section{Caso clínico}

Mujer de 74 años sin hábitos tóxicos y con antecedentes de hipertensión arterial, dislipemia, hipotirodisimo primario y enfermedad renal crónica de etiología no filiada (cuatro años antes presentaba: creatinina plasmática $1.4 \mathrm{mg} / \mathrm{dl}$ y filtrado glomerular estimado (FGE) $39 \mathrm{ml} / \mathrm{min} / 1,73 \mathrm{~m}^{2}$ obtenido por MDRD-4). Desde hacía años recibía tratamiento con telmisartán, simvastatina y levotiroxina. Un mes antes de acudir inició la toma de amlodi-

\begin{abstract}
Multiple drugs can cause non-cardiogenic acute pulmonary edema. Most of the time, establishing this causal relationship is very difficult due to low reported incidence of drug adverse effects and non specific symptoms. Peripheral edema is a common side effect of amlodipine; but only two cases of pulmonary edema caused by amlodipine have been published in the literature and both occurred in the context of drug overdose. We describe a unique case of pulmonary edema probably induced by amlodipine at therapeutic doses and we discuss the possible pathophysiological mechanism.
\end{abstract}

Key words: Amlodipine. Pulmonary edema. Adverse effects.

pino (10 mg/día), notando a los 10 días la aparición de edema progresivo en miembros inferiores y disnea que evolucionó hasta hacerse de mínimos esfuerzos. Su médico asoció furosemida 20 $\mathrm{mg} / \mathrm{día}$ durante la última semana sin mejoría significativa, motivo por el que acudió a urgencias. Además refería ortopnea sin disnea paroxística nocturna y oliguria sin dolor torácico, palpitaciones, fiebre 0 clínica de infección respiratoria. La presión arterial era de 124/72 mm Hg, estaba afebril, el pulso era de 72 latidos por minuto, la frecuencia respiratoria era de 20 respiraciones por minuto y la saturación de oxígeno en aire ambiente era de un 99\%. La coloración cutánea era normal, no presentaba clara ingurgitación venosa yugular, la auscultación cardíaca era normal y en la auscultación pulmonar destacaban crepitantes basales bilaterales. Presentaba edema maleolar simétrico que se extendían hasta rodillas. El resto del examen físico era normal.

El hemograma, estudio básico de coagulación, glucosa, colesterol, triglicéridos, proteínas totales, albúmina, bioquímica hepática, proteína $C$ reactiva, anticuerpos antinucleares, proteinograma y gases en sangre arterial fueron normales. Destacaba: urea 100 $\mathrm{mg} / \mathrm{dl}$, creatinina 2,2 mg/dl, FGE $23,1 \mathrm{ml} / \mathrm{min} / 1,73 \mathrm{~m}^{2}$, tirotropina $14,2 \mathrm{mU} / \mathrm{l}$ y tiroxina libre $1,1 \mathrm{ng} / \mathrm{dl}$. El sedimento urinario y la proteinuria en 24 horas fueron normales. La radiografía de tórax realizada en urgencias mostraba discreto aumento de la silueta cardiovascular, derrame pleural bilateral, lesiones pulmonares bilaterales compatibles con edema y aumento de densidad de aspecto nodular en el lóbulo superior derecho (figura 1). El electrocardiograma era normal. Se sospechó inicialmente insuficiencia cardíaca y edema pulmonar, se pautaron dosis elevadas de furosemida por vía endovenosa, se suspendió amlodipino y se procedió al ingreso. En el ecocardiograma transtorácico se objetivó: ventrículo izquierdo (VI) de tamaño y función sistólica normal con fracción de eyección (FE) de 59,7\% y grosor en telediástole de $11 \mathrm{~mm}$ (tanto a nivel del tabique interventricular como de la pared posterior), flujo transmitral pseudonormal, aurícula izquier- 
Figura 1. Hallazgos compatibles con edema pulmonar. Lesión nodular en el lóbulo superior derecho

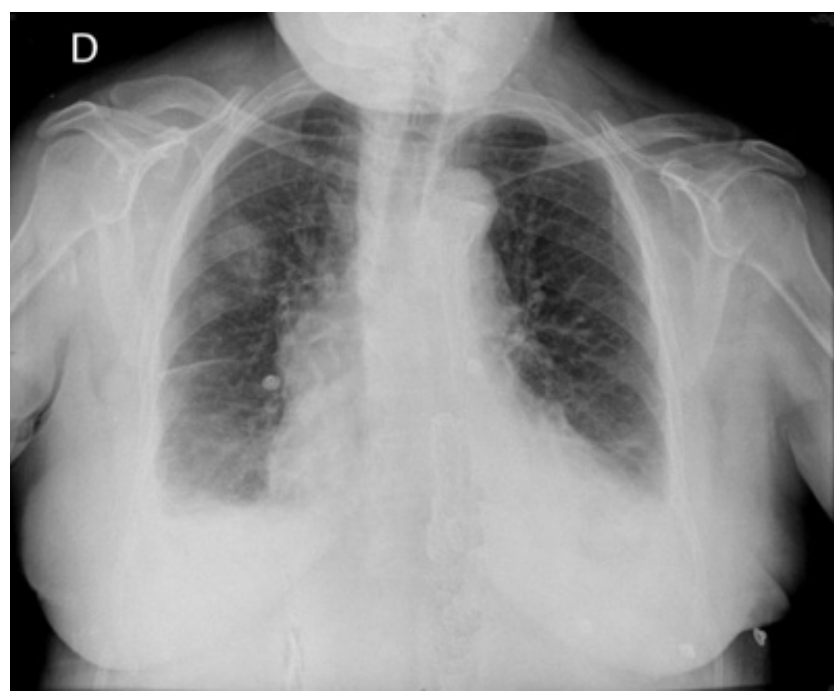

Figura 2. Radiografía de control previa al alta

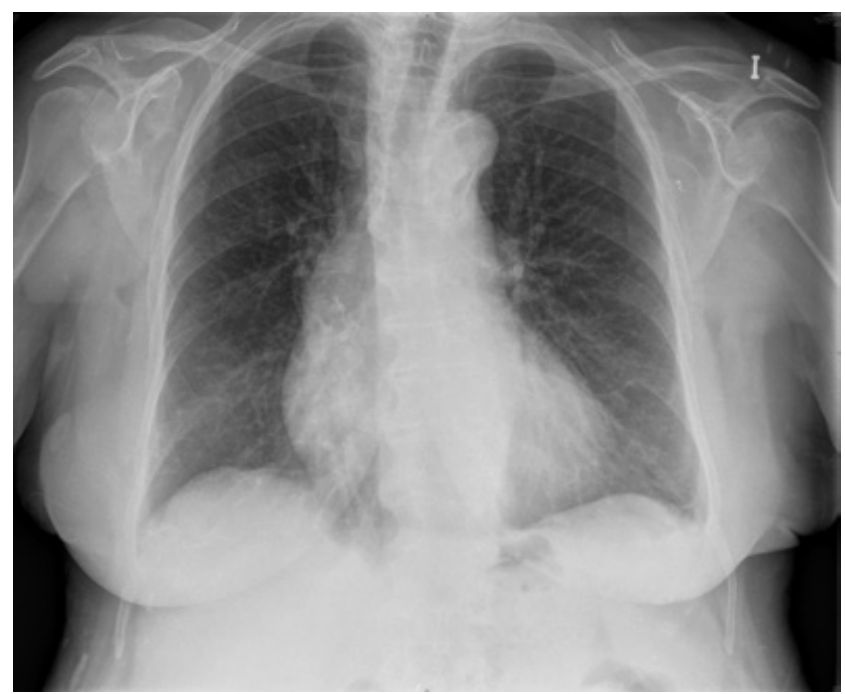

da $3,5 \mathrm{~cm}$, relación entre la onda $E$ y onda $A(E / A)$ igual a 1 , relación entre la velocidad pico de la onda $\mathrm{E}$ mitral y la velocidad $\mathrm{E}$ del anillo lateral mitral (E/e') igual a 11, ventrículo derecho normal, anillo valvular mitral de aspecto levemente calcificado, ausencia de hipertensión pulmonar y vena cava inferior de tamaño normal. La ecografía abdominal no mostraba alteraciones relevantes. En los días siguientes experimentó muy buena evolución: desaparición de la disnea, de los signos de congestión pulmonar y del edema periférico. La radiografía de tórax realizada antes del alta hospitalaria fue normal (Figura 2). Meses más tarde la paciente persistía asintomática.

\section{Discusión}

El edema pulmonar es consecuencia del movimiento de exceso de líquido a los alvéolos como resultado de una alteración en al menos una de las fuerzas de Starling. A dife- rencia del edema pulmonar de origen cardiogénico (EPC), que es causado por un aumento de la presión hidrostática vascular, el edema pulmonar no cardiogénico (EPNC), denominado también daño pulmonar agudo o síndrome de distrés respiratorio agudo ${ }^{5}$, es consecuencia de cambios en la permeabilidad de la membrana capilar pulmonar 6 . Puede resultar difícil de distinguir el EPC y el EPNC debido a sus manifestaciones clínicas similares y conocer la causa tiene importantes implicaciones para el tratamiento ${ }^{5}$.

Entre las entidades clínicas asociadas al EPNC se incluyen: aspiración gástrica, inhalación de toxinas, contusión pulmonar, infección pulmonar difusa, sepsis, reacciones transfusionales sanguíneas, exposición a grandes alturas, insultos neurogénicos, pancreatitis, traumatismos graves y fármacos ${ }^{5}$. Entre los numerosos fármacos implicados destacan: opiáceos, tocolíticos, salicilatos, hidroclorotiacida, protamina, ciclosporina, antidepresivos tricíclicos, amiodarona, alcaloides de la vinca y bleomicina ${ }^{7}$.

Solo se han publicado casos de edema pulmonar asociado a amlodipino empleado a dosis terapéutica en un trabajo, aunque la población estudiada padecía una insuficiencia cardíaca severa ${ }^{2}$, antecedente que no presentaba la paciente que presentamos. La sintomatología y hallazgos en la exploración física y radiografía de tórax hicieron que se sospechase inicialmente una insuficiencia cardíaca. No se determinaron los niveles plasmáticos de péptido natriurético cerebral (BNP). Se ha sugerido que un valor de BNP < 200 pg/ml si el FGE es inferior a $60 \mathrm{ml} / \mathrm{min} / 1,73 \mathrm{~m}^{2}$ excluiría una insuficiencia cardíaca ${ }^{8}$. Aunque los hallazgos en la radiografía de tórax pueden ayudar a diferenciar el EPC del EPNC su precisión diagnóstica es limitada ${ }^{5}$.

Los hallazgos ecocardiográficos no apoyaban una insuficiencia cardíaca con disfunción sistólica como explicación del cuadro clínico. Ante la buena evolución clínica y radiológica (incluida la desaparición de la lesión nodular pulmonar del lóbulo superior derecho, que interpretamos como tumor fantasma) no fue preciso determinar la presión de enclavamiento pulmonar (PCWP), considerada la prueba de referencia para diferenciar EPC de EPNC. Sin embargo, actualmente mediante Doppler tisular, si la FE del VI es normal, se considera que con una relación E/e' $\geq 13$ existe aumento de presión de llenado del VI ${ }^{9}$. En nuestro caso, la relación E/e' se situó en una zona intermedia, dato significativo en contra de EPC aunque no concluyente. El hallazgo de un flujo transmitral pseudonormal podría sugerir una insuficiencia cardíaca diastólica, aunque la relación E/e' obtenida, la ausencia de hipertrofia ventricular izquierda significativa, el tamaño normal de la aurícula izquierda, la ausencia de hipertensión pulmonar y la no existencia de un factor clínico desencadenante claro la hacían improbable. No se realizó coronariografía para valorar la existencia de una posible enfermedad coronaria debido a la baja sospe- 
cha clínica (ausencia de dolor torácico, no cambios electrocardiográficos ni ecocardiográficos sugestivos) y a la excelente evolución. Además, en este contexto resultaría sorprendente que si fuese la enfermedad coronaria la causa del edema pulmonar, aconteciese precisamente a los pocos días de haberse iniciado el tratamiento con amlodipino, fármaco conmúnmente empleado en el tratamiento de la cardiopatía isquémica.

Está descrito que el hipotiroidismo subclínico puede asociarse con un mayor riesgo de insuficiencia cardíaca ${ }^{10}$. Este trabajo, sin embargo, contaba con ciertas limitaciones: no disponer de determinación de tiroxina libre en algunos pacientes, la posible clasificación incorrecta de los episodios de insuficiencia cardíaca y el haberse realizado en pacientes mayores de 70 años (al igual que el caso que se presenta) con alta prevalencia de ambas patologías. Además, no se hacía referencia a la posible relación entre hipotirodismo subclínico y edema pulmonar ya que tampoco se mencionaba la presencia 0 ausencia de hallazgos radiológicos compatibles con el mismo. En definitiva, consideramos improbable que el hipotiroidismo subclínico explicase el cuadro clínico.

El edema periférico tampoco se justificaba por una hepatopatía, síndrome nefrótico, malnutrición ni enteropatía pierde-proteínas. Por tanto, se consideró por exclusión y aparente relación temporal la posibilidad de amlodipino como causa del edema periférico y pulmonar.

El edema periférico asociado a las dihidropiridinas se atribuye a un aumento del fluido filtrado desde el compartimento vascular al intersticial debido a un aumento de la presión hidrostática intracapilar, causado por una inhibición más pronunciada del tono vascular precapilar que en el poscapilar ${ }^{11}$.

El edema pulmonar en pacientes tratados con amlodipino no tiene un mecanismo fisiopatológico tan bien definido. Estudios en animales demostraron que los antagonistas del calcio aumentan el fluido filtrado y la permeabilidad a la albúmina en las arterias de diferentes órganos, como pul- mones, testículos, cerebro y corazón ${ }^{12}$. En el caso descrito, Ios pulmones podrían haberse afectado primariamente.

En base al algoritmo de Naranjo ${ }^{13}$ para la evaluación de la causalidad de una reacción adversa a medicamentos (RAM), correspondería a una RAM posible. El tratamiento consistió en la suspensión de amlodipino y en medidas de soporte. Se notificó electrónicamente el caso al Sistema Español de Farmacovigilancia de Medicamentos de uso Humano (SEFV-H).

En conclusión, en la historia de los pacientes con EPNC es importante considerar cualquier exposición a fármacos e incluir en el diagnóstico diferencial a amlodipino como posible agente causal.

\section{Bibliografía}

1. Ficha técnica. Amlodipino. Agencia Española de Medicamentos y Productos Sanitarios. Ministerio de Sanidad y Consumo. Septiembre 2012. Disponible en http:// www.aemps.gob.es.

2. Packer M, O'Connor CM, Ghali JK, Pressler ML, Carson PE, Belkin RN, et al. Effect of amlodipine on morbidity and mortality in severe chronic heart failure. Prospective Randomized Amlodipine Survival Evaluation Study Group. N Engl J Med 1996;335(15):1107-1114.

3. Stanek EJ, Nelson CE, DeNofrio D. Amlodipine overdose. Ann Pharmacother 1997;31:853-6.

4. Saravu K, Balasubramanian R. Near-fatal amlodipine poisoning. J Assoc Physicians India. 2004;52:156-7.

5. Ware LB, Matthay MA. Clinical practice. Acute pulmonary edema. N Engl J Med 2005;353:2788.

6. Perina DG. Noncardiogenic pulmonary edema. Emerg Med Clin North Am 2003;21:385-393.

7. Reed CR, Glauser FL. Drug-induced noncardiogenic pulmonary edema. Chest 1991;100:1120-4.

8. Silver MA, Maisel A, Yancy CW, McCullough PA, Burnett JC Jr, Francis GS, et al. BNP Consensus Panel 2004: A clinical approach for the diagnostic, prognostic, screening, treatment monitoring, and therapeutic roles of natriuretic peptides in cardiovascular diseases. Congest Heart Fail 2004;10(5 Suppl 3):1-30.

9. Nagueh SF, Appleton CP, Gillebert TC, Marino PN, Oh JK, Smiseth OA, et al. Recommendations for the evaluation of left ventricular diastolic function by echocardiography. J Am J Echocardiogr 2009;22:107-23.

10. Rodondi N, Newman AB, Vittinghoff E, de Rekeneire N, Satterfield S, Harris TB, Bauer DC. Subclinical hypothyroidism and the risk of heart failure, other cardiovascular events, and death. Arch Intern Med 2005;165:2460-6.

11. Gustafsson D. Microvascular mechanisms involved in calcium antagonista edema formation. J Cardiovasc Pharmacol 1987;10(Suppl 1):S121-S131.

12. Lacolley $P$, Poitevin $P$, Koen R, Levy Bl. Different effects of calcium antagonists on fluid filtration of large arteries and albumin permeability in spontaneously hypertensive rats. J Hypertens 1998;16:349-55.

13. Naranjo CA, Busto U, Sellers EM, Sandor P, Ruiz I, Roberts EA, et al. A method for estimating the probability of adverse drug reactions. Clin Pharmacol Ther 1981;30:239-45. 